Version of Record: https://www.sciencedirect.com/science/article/pii/S1297319X19300144

Manuscript_b56af48b2b606b163fb34216e7cecc4a

\title{
Cryoglobulinemia: an update in 2019
}

\section{Anne Claire Desbois ${ }^{1,2,3}$, Patrice Cacoub ${ }^{1,2,3}$, David Saadoun ${ }^{1,2,3}$}

${ }^{1}$ Sorbonne Universités, UPMC Univ Paris 06, INSERM, UMR S 959, Immunology-

Immunopathology- Immunotherapy (I3) ; F-75005, Paris, France

${ }^{2}$ Biotherapy (CIC-BTi) and Inflammation-Immunopathology-Biotherapy Department (DHU i2B), Hôpital Pitié-Salpêtrière, AP-HP, F-75651, Paris, France

${ }^{3}$ AP-HP, Groupe Hospitalier Pitié-Salpêtrière, Department of Internal Medicine and Clinical Immunology, F-75013, Paris, France, Centre national de références Maladies Autoimmunes et systémiques rares et Maladies Autoinflammatoires rares

Correspondence: David Saadoun, Département de Médecine Interne et d’Immunologie clinique, Hôpital Pitié-Salpétrière, Batiment La Rochefoucauld, 83 boulevard de l'hôpital, 75013 Paris, FRANCE

Tel : +33142178009; Fax: +33142178033

E-mail: david.saadoun@aphp.fr 


\begin{abstract}
Cryoglobulinemia is defined as the persistent presence in serum of abnormal immunoglobulins (Igs) that precipitate at low temperatures and dissolve again upon warming. Cryoglobulins may be composed only of a monoclonal Ig (simple type I cryoglobulinemia), of a monoclonal Ig bound to the constant domain of polyclonal Ig heavy chains (mixed type II cryoglobulinemia), or only of polyclonal Igs (mixed type III cryoglobulinemia). The manifestations of type I cryoglobulinemia are often related to intravascular obstruction, whereas those seen in the mixed cryoglobulinemias often originate in true immune complex-mediated vasculitis. The main clinical manifestations affect the skin (purpura, necrotic ulcers), joints, peripheral nervous system, and kidneys (membranoproliferative glomerulonephritis). Patients with type I cryoglobulinemia should be investigated for hematological malignancies (myeloma and B-cell lymphoma). Hepatitis $\mathrm{C}$ is the main diagnosis to consider in patients with mixed cryoglobulinemia, followed by connective tissue disease and B-cell non-Hodgkin's lymphoma. The treatment depends mainly on the cause of the cryoglobulinemia. For instance, hepatitis $\mathrm{C}$ virus (HCV) eradication is in order in patients with $\mathrm{HCV}$-associated cryoglobulinemia vasculitis, and the underlying hematological malignancy must be treated in patients with type I cryoglobulinemia.
\end{abstract}

Keywords: Vasculitis. Cryoglobulin. Hepatitis C. Hematological disease. 


\section{What is cryoglobulinemia?}

A cryoglobulin is an abnormal immunoglobulin (Ig) that precipitates at low temperature then dissolves again when the temperature is increased. This definition distinguishes cryoglobulins from cryoproteins and cold agglutinins. Cryoglobulinemia is defined as the persistent presence of cryoglobulins in serum.

\subsection{Clinical and immunological classification of the cryoglobulinemias}

Brouet's classification is the most widely used for distinguishing among the various types of cryoglobulinemia [1]. The chemical and immunological features of the cryoglobulins are used to define three types. Type I cryoglobulinemia or simple cryoglobulinemia is characterized by a single monoclonal Ig, which is usually an $\operatorname{IgM}$ or IgG. In type II cryoglobulinemia, polyclonal Igs form immune complexes with one or more monoclonal Igs. The most common form combines a monoclonal IgM and polyclonal IgGs (mixed monoclonal cryoglobulinemia). When the Ig components are separated, none precipitates at low temperatures. The monoclonal IgM has rheumatoid factor activity and binds to the $\mathrm{Fc}$ of the IgGs, which, in turn, are bound to an antigen (e.g., the hepatitis $\mathrm{C}$ virus $[\mathrm{HCV}]$ genome). These numerous chemical interactions result in strong stability of the IgGIgM complex. Finally, type III cryoglobulinemia involves only polyclonal Igs. Polyclonal IgMs and IgGs may form immune complexes (mixed polyclonal cryoglobulinemia). Type III cryoglobulins are common and frequently detectable in low levels in healthy individuals. In the absence of other abnormalities, their clinical significance is unclear.

One of the benefits of this immunochemical classification is that it provides guidance for both the etiological diagnosis and the treatment (Table 1). Type I cryoglobulinemia, 
which accounts for $10 \%-15 \%$ of cases of symptomatic vasculitis, is often associated with hematological malignancies such as myeloma or B-cell lymphoma or with monoclonal gammopathy of undetermined significance (MGUS). Mixed cryoglobulinemia (80\%-85\%), in contrast, is associated with infectious diseases, notably chronic HCV infection; B-cell malignancies; and autoimmune diseases such as Sjögren’s syndrome and lupus. Mixed cryoglobulinemia remains idiopathic in $15 \%$ of cases.

\subsection{Detection of cryoglobulins and diagnosis of cryoglobulinemia}

Several precautions are important to avoid false-negative results from cryoglobulin detection tests. To avoid premature cryoprecipitation, the blood sample must be kept at $37^{\circ}$ during transport to the laboratory then during centrifugation. Cryoglobulins precipitate below $37{ }^{\circ} \mathrm{C}$. After centrifugation, the serum sample is stored at $4{ }^{\circ} \mathrm{C}$ for 8 days to ensure the detection of delayed cryoprecipitation. The cryoprecipitate is dissolved by increasing the temperature. After purification, immunoelectrophoresis is performed to identify the type of cryoglobulin as I, II, or III, as described above. The cryoprecipitate is quantified using immunofixation or Western blotting. The severity of the clinical manifestations does not consistently correlate with the serum concentration of cryoglobulin, although symptomatic patients have higher concentrations on average than do asymptomatic patients [2]. After lengthy debates, it was determined that a concentration above $50 \mathrm{mg} / \mathrm{L}$ is considered abnormal.

Cryoglobulin detection can be technically difficult due to the considerable thermal instability of cryoglobulins, which precipitate readily if the temperature of the blood sample falls below $37^{\circ}$ before processing at the laboratory. Consequently, evaluation for other laboratory features that suggest cryoglobulinemia is important. Although inconsistently present, several complement abnormalities are fairly specific, such as decreases in early 
components $(\mathrm{C} 1 \mathrm{q}, \mathrm{C} 2$, and $\mathrm{C} 4)$ and in $\mathrm{CH} 50$ and increased levels of the late components (C5 and C9) and of C1 inhibitor; the C3 level is usually normal. Rheumatoid factor activity is common in mixed cryoglobulinemia but is rarely found in type I cryoglobulinemia (Table 2). Electrophoresis and immunoelectrophoresis show either polyclonal hypergammaglobulinemia or a monoclonal peak.

Mixed cryoglobulinemic vasculitis manifests typically as vascular purpura, cryoglobulinemia, and low C4 levels. However, patients with true cryoglobulinemic vasculitis may have negative tests for cryoglobulins.

Liver test abnormalities are extremely common in patients with mixed cryoglobulinemia. About $50 \%$ to $70 \%$ have high transaminase and alkaline phosphatase levels. These abnormalities are related to chronic HCV infection in most cases and far less often to chronic hepatitis B virus (HBV) infection.

The presence of a cryoglobulin can alter the results of standard blood tests such as the protein level and the erythrocyte sedimentation rate. The gammaglobulin level may be spuriously decreased due to precipitation of the cryoglobulin.

Cryoglobulinemia is associated with nonnecrotizing leukocytoclastic vasculitis involving small vessels (arterioles, capillaries, and venules) and medium-sized vessels. The inflammatory infiltrate composed predominantly of lymphocytes and monocytes, with few neutrophils, is present mainly around the blood vessels, where it forms sheaths but shows little or no tendency to invade the vessel walls. However, $20 \%$ of patients have necrotizing vasculitis with fibrinoid necrosis preferentially involving the medium-sized vessels in a presentation similar to that of polyarteritis nodosa.

\section{Cryoglobulinemic vasculitis: Clinical presentation (Figure 1)}


Cryoglobulinemia can cause vasculitides that belong to the group of systemic immune-complex-mediated vasculitis. Vasculitis is most common in mixed cryoglobulinemia. Females are affected more often than males, with a female/male ratio of 2/1. Symptom onset is in the fourth or fifth decade, with no differences across ethnic groups. General symptoms are common, notably severe fatigue. Unexplained fever with or without weight loss may be a feature. Table 1 lists the main clinical manifestations and their frequency in each type of cryoglobulinemia.

\subsection{Vascular purpura}

Vascular purpura is often the inaugural manifestation. The lower limbs are affected first but the lesions may then extend to the abdomen. The purpura follows an intermittent relapsing course. The lesions are infiltrated non-pruriginous petechiae or papules. Necrosis may develop, notably in patients with type I cryoglobulinemia. Distal necrosis at the upper or lower limbs is also most common in type I cryoglobulinemia. Cold-induced symptoms such as Raynaud's phenomenon occur in $25 \%$ of patients overall, with higher rates in patients who have type I cryoglobulinemia.

Cold urticaria is a chronic systemic non-pruriginous urticarial rash with plaques that remain unchanged for more than 24 hours. The trigger is exposure to cold. The rash can be induced by placing an ice cube on the forearm.

\subsection{Joint involvement}

The joint manifestations (50\%-75\%) often consist of non-migratory pain that predominantly involves the hands and knees; the elbows and ankles are less often affected. The distribution is bilateral and symmetric. There are no joint deformities. True arthritis is less common and does not cause joint destruction. Spinal involvement is even less frequent. 


\subsection{Renal involvement}

Renal involvement is usually a late manifestation. Proteinuria and microscopic hematuria are the main manifestations, although renal failure may develop. Atypical nephrotic syndrome or acute nephritic syndrome occurs in some patients. Hypertension often develops shortly after the onset of the renal manifestations.

The renal biopsy evidences diffuse global membranoproliferative glomerulonephritis with mesangial proliferation, which may have a nodular appearance. Extra-capillary proliferation may be present. Histological findings of adverse prognostic significance include amorphous eosinophilic intraluminal thrombi and vasculitis affecting the small and medium-size vessels ( $25 \%$ ) with fibrinoid necrosis of the vessel walls. Immunofluorescence shows subendothelial and intraluminal deposits of immunoglobulins identical to those present in the cryoprecipitate; C3 is found only in the subendothelial deposits. By electronic microscopy, the subendothelial and intraluminal deposits are seen to be organized into microtubules measuring 10 to $25 \mathrm{~nm}$ in diameter.

\subsection{Neurological involvement}

The peripheral nervous system is the main target. Distal sensory or sensorimotor polyneuropathy predominating at the lower limbs occurs in two-thirds of patients and mononeuritis multiplex in one-third of patients. Common inaugural symptoms include disorders in superficial sensation, with neuropathic pain and paresthesia. Motor loss is less common, delayed by a few months to a few years, and sets in gradually; the anterolateral leg compartments are preferentially affected, often asymmetrically [3].

\section{Other, rare manifestations $(<5 \%)$}




\subsection{Gastrointestinal involvement}

Patients may experience abdominal pain, which may simulate a surgical condition. Gastrointestinal tract bleeding or perforation may occur. These gastrointestinal manifestations are due to distal vasculitis involving the mesenteric arterioles and capillaries.

\subsection{Involvement of the central nervous system}

Vasculitis of the brain, although rare, is probably underestimated [4]. The manifestations include acute or subacute neurological deficits, headaches, seizures, cranial nerve impairments and, in some cases, cerebrovascular events. Histological proof of cerebral vasculitis is only very rarely obtained. The presumptive diagnosis rests on the findings from imaging studies, notably magnetic resonance imaging angiography or computed tomography. One or more ischemic lesions may be visible, as well as distal obstruction or irregularities in vessel diameter. The cerebrospinal fluid is usually normal, although pleocytosis and protein level elevation have been reported [5]. One suggested mechanism is ischemia related to intravascular cryoglobulin precipitation within small brain vessels free from vasculitis lesions [4].

\subsection{Cardiac involvement}

Cardiac involvement is rare but associated with increased mortality. Microvascular disease develops and may cause severe heart failure, which is among the causes of death in patients with cryoglobulinemia. The postmortem examination shows necrotizing vasculitis of the coronary arterioles. Myocardial infarction due to lesions of the large coronary arteries is also possible. Acute pericarditis is more common and rarely responsible for complications. 


\subsection{Pulmonary involvement}

Lung disease is extremely rare. Bronchiolitis obliterans organizing pneumonia and alveolar hemorrhage syndromes are probably due to vasculitis and o lymphocytic alveolitis.

\section{Pathophysiology}

The mechanism underlying the formation of cryoprecipitates is unclear but depends on numerous parameters including the Ig level, $\mathrm{pH}$, ionic force, and temperature, as well as the electrical charge, which is governed by the amino acid sequences and sugar moieties contained in the immunoglobulin.

Ischemic lesions may be related to vascular obstruction by cryoglobulin precipitates, notably in type I cryoglobulinemia. Mixed cryoglobulinemia causes true immune complexmediated vasculitis. Why symptomatic vasculitis does not occur consistently is unclear. Recent studies indicate that the development of lesions is heavily dependent on the physicochemical properties of the Igs, such as stereotactic properties and heavy-chain glycosylation. Depending on their physicochemical properties, Igs vary in their propensity to form immune complexes, precipitate, and induce an inflammatory response (via recruitment of complement and of the macrophage Fc receptor) [6]. More specifically, differences in solubility and rigidity influence the tendency to form immune complexes, and some Igs tend to undergo cleavage, which limits the size of the immune complexes.

In chronic hepatitis $\mathrm{C}$, the $\mathrm{HCV}$ envelope glycoproteins $\mathrm{E} 1$ and $\mathrm{E} 2$ help the virus enter into the hepatocytes and lymphocytes, possibly via the CD81 cell receptor [7]. Chronic HCV infection induces persistent stimulation of the intrahepatic and circulating B cells. In patients with HCV-related cryoglobulinemia, several studies have demonstrated oligoclonal 
or monoclonal expansion of the $\operatorname{IgM}^{+}, \operatorname{IgD}^{+} \mathrm{CD} 21^{\text {low }}$ memory B cells. The expanded B-cell population is characterized by a distinctive repertoire, with predominance of certain clones, notably VH1-69, which produce an Ig with rheumatoid factor activity, thereby leading to the formation of a cryoglobulin [8,9]. The chronic antigen stimulation results in the gradual emergence of B-cell clones that produce polyclonal IgMs (type III cryoglobulin) initially, then oligoclonal IgMs (type II/III cryoglobulin), and finally a monoclonal IgM (type II cryoglobulinemia) [2]. In patients with mixed cryoglobulinemia, HCV proteins have been identified in skin (E2 and core proteins) and kidney (core protein) biopsies and genomic HCV RNA in nerve tissues in patients with HCV-related mixed cryoglobulinemia [10].

Although all B-cell non-Hodgkin's lymphoma subtypes have been reported in association with $\mathrm{HCV}$, the most common are marginal zone lymphoma and diffuse large Bcell lymphoma resulting from the transformation of low-grade lymphoma [11,12]. In patients with symptomatic cryoglobulinemia, the risk of developing lymphoma may be increased 35fold compared to the general population [13]. The current model for HCV-related lymphoma involves an additional oncogenic event affecting a B-cell clone previously selected via chronic stimulation by HCV antigens (see above).

\section{Causes of cryoglobulinemias}

The vast number of varied conditions associated with the production of cryoglobulins can be roughly classified into three categories: chronic hepatitis $\mathrm{C}$ and other infections, autoimmune diseases (Sjögren's syndrome and lupus), and B-cell lymphoid malignancies (Box 1 and Figure 2).

\subsection{Type I cryoglobulinemia}


Simple monoclonal (type I) cryoglobulinemia is associated with B-cell malignancies, notably Waldenström macroglobulinemia and myeloma, and with MGUS. The ability to form cryoprecipitates is a feature of the monoclonal protein. The manifestations are often related to small-vessel obstruction by the monoclonal Ig precipitates. Monoclonal IgM cryoglobulinemia suggests Waldenström macroglobulinemia or another type of lymphoma and monoclonal IgG cryoglobulinemia myeloma or MGUS. The clinical manifestations of cryoglobulinemia may antedate the diagnosis of Waldenström macroglobulinemia by several months or years.

Type I cryoglobulinemia may also occur in patients with non-Hodgkin's lymphoma or B-cell chronic lymphocytic leukemia, although mixed type II cryoglobulinemia is more common in these conditions.

\subsection{Type II and III mixed cryoglobulinemia}

$\mathrm{HCV}$ infection is the leading cause of mixed (type II and III) cryoglobulinemia, with $70 \%$ to $90 \%$ of cases being due to this virus. Mixed cryoglobulinemia is found in $25 \%$ to $30 \%$ of patients with hepatitis $\mathrm{C}$ but is generally asymptomatic. Thus, symptomatic vasculitis develops in only $10 \%$ to $15 \%$ of patients with hepatitis C. In studies conducted in various geographic regions, the prevalence of serum anti-HCV antibodies among patients with mixed cryoglobulinemia ranged from $56 \%$ to $95 \%[\mathbf{1 4 , 1 5}]$. In the vast majority of HCV-positive patients, the viral RNA was found in the serum and within the cryoprecipitate.

About $10 \%$ to $30 \%$ of cases of mixed cryoglobulinemia (types II and III) are not related to $\mathrm{HCV}$. The many causes include other infections, B-cell lymphoid malignancies, and autoimmune diseases (Table I). In a recent study of 242 patients with noninfectious cryoglobulinemia vasculitis, connective tissue disease (Sjögren's syndrome, followed by lupus and scleroderma) was diagnosed in $30 \%$ of cases and a B-cell malignancy (chiefly 
marginal zone lymphoma, B-cell non-Hodgkin's lymphoma, and lymphoplasmacytic lymphoma) in $22 \%$ of cases, with the remaining $48 \%$ of patients being classified as having idiopathic cryoglobulinemia [16]. Mixed type II or III cryoglobulinemia is not uncommon in patients with primary Sjögren's syndrome but long remains asymptomatic. The development of vasculitis with cutaneous and peripheral nervous system involvement should prompt an evaluation for lymphoma.

Table 2 lists the main differences in the clinical and laboratory features of simple (type I) and mixed (types II and III) cryoglobulinemia.

\section{Prognosis}

The course and outcome of cryoglobulinemia varies considerably across individuals. The prognosis depends chiefly on the severity of the organ involvement and more specifically of the renal, gastrointestinal, cardiac, and/or central nervous system manifestations An underlying hematological malignancy is also a major prognostic factor. In a retrospective study of 242 patients with noninfectious symptomatic mixed cryoglobulinemia, compared to the type III subgroup, the type II subgroup was characterized by higher prevalences of purpura, renal involvement, and peripheral neurological involvement; higher cryoglobulin concentrations; and lower C3 and C4 concentrations [16].

Among the manifestations of cryoglobulinemic vasculitis, the gastrointestinal and myocardial involvements lead to the highest risk of death. Prognostic factors in early caseseries studies included age older than 65 years and renal involvement. In keeping with these data, a more recent study of noninfectious mixed cryoglobulinemic vasculitis found poorer outcomes in patients with pulmonary involvement, gastrointestinal involvement, a creatinine 
clearance below $60 \mathrm{~mL} / \mathrm{min}$, and age older than 65 years [16]. Survival rates after 1, 2, 5, and 10 years were $91 \%, 89 \%, 79 \%$, and $65 \%$, respectively.

The main causes of death in patients with HCV-related cryoglobulinemic vasculitis are infections related to immunosuppressive therapy, liver cirrhosis, cardiovascular involvement, and severe renal dysfunction. The main adverse prognostic factors reported to date are severe hepatic fibrosis (METAVIR score $\geq 3$ ) and involvement of the central nervous system, kidneys, or heart (hazard ratios: 2.7, 1.9, and 4.2, respectively) [17].

\section{Treatment strategy}

\subsection{Type I Cryoglobulinemia}

In patients with symptomatic cryoglobulinemia type I, the treatment strategy focuses on the underlying hematological malignancy. Thus, lymphoma requires combination chemotherapy and myeloma treatment with drugs such as bortezomib, thalidomide, lenalidomide, or alkylating agents [18]. Autologous bone marrow transplantation may be in order in myeloma-related cryoglobulinemia. IgG MGUS, which indicates plasma cell proliferation, is treated with those myeloma drugs that target the plasma cells, whereas rituximab is generally preferred for IgM MGUS, which indicates lymphoplasmacytic proliferation [19]. Plasma exchange therapy is warranted in selected patients with severe renal involvement or extensive leg necrosis [21]. Exposure to low temperatures exacerbates cryoglobulin formation and should therefore be avoided [20,21].

\subsection{Mixed cryoglobulinemia}

7.2.1. HCV-related cryoglobulinemia vasculitis 
Sustained suppression of HCV replication is associated with a significantly higher rate of complete clinical remission in patients with cryoglobulinemia vasculitis [22]. In studies reported in 2011 and 2013, antiviral therapy combining pegylated interferon (PegIFN) and ribavirin for 12 months produced a sustained virological response in $50 \%$ to $60 \%$ of patients with HCV-related vasculitis $(17,18)$. Virological relapses after an initial response were usually accompanied with relapsing vasculitis (19). When PegIFN and ribavirin were combined with direct-acting antivirals, i.e., an NS3/4A protease inhibitor such as boceprevir or telaprevir, the rate of sustained virological response increased to $65 \%-70 \%$ in patients infected with HCV genotype $1[13,14]$. Prolonged therapy (48 weeks) was required however, and the rate of serious adverse events was $47 \%$ [13].

The introduction of other direct-acting antivirals has radically transformed the management of HCV-related cryoglobulinemic vasculitis. All these new drugs allow substantially shorter treatments, without interferon, and produce sustained virological response rates greater than $95 \%$ with relatively few adverse effects. In the earliest open-label prospective study, 24 patients with HCV-related cryoglobulinemic vasculitis, including 50\% with HCV genotype 1 and 50\% with cirrhosis of the liver, combined sofosbuvir and ribavirin therapy induced a complete clinical remission in $87.5 \%$ of cases with a sustained virological response 12 weeks after treatment discontinuation in $74 \%$ of patients [15].

Other studies of these new antivirals showed similar results, with high rates of virological response and clinical vasculitis remission [23-25]. In 44 consecutive patients with vasculitis related to the HCV (genotypes 1, 2, 3, and 4 in 23, 13, 5, and 3 patients, respectively), the treatments used were sofosbuvir with ribavirin alone $(n=18)$ or with simeprevir $(n=12)$, ledipasvir $(n=10)$, or daclatasvir $(n=4)$, combined in 9 patients with ribavirin [17]. Low-dose rituximab was given to 2 patients with severe vasculitis. After 12 and 24 weeks, the HCV was undetectable and all patients had a clinical vasculitis response. 
The mean Birmingham Vasculitis Activity Score decreased from 5.41 at baseline to 2.35 at week $4,1.39$ at week 12 , and 1.27 at week 24 . The mean cryocrit value fell from $7.2 \%$ at baseline to $1.8 \%$ at week 24 . Adverse drug events were recorded in $59 \%$ of patients but all were minor except 1 case of ribavirin-related anemia requiring blood transfusion.

Despite the marked efficacy of antivirals on the symptoms of HCV-related cryoglobulinemic vasculitis, immunosuppressive drugs remain a valid option in selected patients. Immunosuppressant therapy is indispensable in patients with severe vasculitis manifestations such as severe kidney dysfunction, skin necrosis, and/or involvement of the gastrointestinal tract or central nervous system. Rituximab has demonstrated greater efficacy than conventional immunosuppressants or placebo therapy [20,21]. In earlier studies, adding rituximab to Peg-IFN and ribavirin shortened the time to clinical remission and increased the renal response and cryoglobulin clearance rates [22,23].

Direct-acting antivirals should be viewed as first-line treatments in patients with HCV-related cryoglobulinemic vasculitis. Selection of the antiviral combination is guided by the clinical manifestations of vasculitis, HCV genotype, and severity of fibrosis. Rituximab targets the clonal B-cell population upstream from cryoglobulin production and is also used to treat severe vasculitis. Plasma exchange is indicated in patients with refractory disease and/or severe organ involvement (rapidly progressive glomerulonephritis, gastrointestinal vasculitis, severe mononeuritis multiplex, or myocarditis) and/or severe cutaneous involvement (extensive ulcers, distal ischemia). Intravenous methylprednisolone and immunosuppressants, notably cyclophosphamide, also deserve consideration in these very severe forms.

\subsubsection{Cryoglobulinemic vasculitis unrelated to HCV}


Treatment at the case is the main focus in patients with symptomatic mixed cryoglobulinemia unrelated to the $\operatorname{HCV}[22,23]$. Infection should be managed with appropriate anti-infectious agents. Rituximab combined with glucocorticoid therapy is the first-line treatment in the event of autoimmune disease [26,27]. Plasma exchange therapy may deserve consideration in patients at the severe end of the clinical spectrum.

In conclusion, cryoglobulinemia is defined as the persistent presence in serum of abnormal Igs that precipitate at low temperatures then dissolve again when the temperature is increased. Cryoglobulins are composed of either a monoclonal Ig (type I), a complex formed by a monoclonal Ig bound to the constant domain of polyclonal Ig heavy chains (type II), or only of polyclonal Igs (type III). Patients with type I cryoglobulinemia should be investigated for a hematological malignancy (myeloma or B-cell lymphoma, notably lymphoplasmacytic lymphoma). Among the main diagnoses to consider in patients with type II or III cryoglobulinemia, $\mathrm{HCV}$ infection is the most common, followed by connective tissue disease and B-cell non-Hodgkin lymphoma. The treatment strategy depends on the cause of the cryoglobulinemia. 


\section{References}

[1] Brouet JC, Clauvel JP, Danon F, Klein M, Seligmann M. Biologic and clinical significance of cryoglobulins. A report of 86 cases. Am J Med 1974;57:775-88.

[2] Sene D, Ghillani-Dalbin P, Thibault V, Guis L, Musset L, Duhaut P, et al. Longterm course of mixed cryoglobulinemia in patients infected with hepatitis $\mathrm{C}$ virus. J Rheumatol 2004;31:2199-206.

[3] Cacoub P, Saadoun D, Limal N, Léger JM, Maisonobe T. Hepatitis C virus infection and mixed cryoglobulinaemia vasculitis: a review of neurological complications. AIDS Lond Engl 2005;19 Suppl 3:S128-134.

[4] Casato M, Saadoun D, Marchetti A, Limal N, Picq C, Pantano P, et al. Central nervous system involvement in hepatitis $\mathrm{C}$ virus cryoglobulinemia vasculitis: a multicenter casecontrol study using magnetic resonance imaging and neuropsychological tests. J Rheumatol $2005 ; 32: 484-8$.

[5] Petty GW, Duffy J, Houston J. Cerebral ischemia in patients with hepatitis C virus infection and mixed cryoglobulinemia. Mayo Clin Proc 1996;71:671-8.

[6] Strait RT, Posgai MT, Mahler A, Barasa N, Jacob CO, Köhl J, et al. IgG1 protects against renal disease in a mouse model of cryoglobulinaemia. Nature 2015;517:501-4. doi:10.1038/nature13868.

[7] Pileri P, Uematsu Y, Campagnoli S, Galli G, Falugi F, Petracca R, et al. Binding of hepatitis C virus to CD81. Science 1998;282:938-41.

[8] Terrier B, Joly F, Vazquez T, Benech P, Rosenzwajg M, Carpentier W, et al. Expansion of functionally anergic CD21-/low marginal zone-like B cell clones in hepatitis C virus infection-related autoimmunity. J Immunol Baltim Md 1950 2011;187:6550-63. doi:10.4049/jimmunol.1102022. 
[9] Charles ED, Brunetti C, Marukian S, Ritola KD, Talal AH, Marks K, et al. Clonal B cells in patients with hepatitis $\mathrm{C}$ virus-associated mixed cryoglobulinemia contain an expanded anergic CD21low B-cell subset. Blood 2011;117:5425-37. doi:10.1182/blood-2010-10312942.

[10] Sansonno D, Cornacchiulo V, Iacobelli AR, Di Stefano R, Lospalluti M, Dammacco F. Localization of hepatitis $\mathrm{C}$ virus antigens in liver and skin tissues of chronic hepatitis $\mathrm{C}$ virus-infected patients with mixed cryoglobulinemia. Hepatol Baltim Md 1995;21:305-12.

[11] Saadoun D, Suarez F, Lefrere F, Valensi F, Mariette X, Aouba A, et al. Splenic lymphoma with villous lymphocytes, associated with type II cryoglobulinemia and HCV infection: a new entity? Blood 2005;105:74-6. doi:10.1182/blood-2004-05-1711.

[12] Mele A, Pulsoni A, Bianco E, Musto P, Szklo A, Sanpaolo MG, et al. Hepatitis C virus and B-cell non-Hodgkin lymphomas: an Italian multicenter case-control study. Blood 2003;102:996-9. doi:10.1182/blood-2002-10-3230.

[13] Monti G, Pioltelli P, Saccardo F, Campanini M, Candela M, Cavallero G, et al. Incidence and characteristics of non-Hodgkin lymphomas in a multicenter case file of patients with hepatitis C virus-related symptomatic mixed cryoglobulinemias. Arch Intern Med 2005;165:101-5. doi:10.1001/archinte.165.1.101.

[14] Cacoub P, Poynard T, Ghillani P, Charlotte F, Olivi M, Piette JC, et al. Extrahepatic manifestations of chronic hepatitis C. MULTIVIRC Group. Multidepartment Virus C. Arthritis Rheum 1999;42:2204-12. doi:10.1002/1529-0131(199910)42:10<2204::AIDANR24>3.0.CO;2-D.

[15] Monti G, Galli M, Invernizzi F, Pioltelli P, Saccardo F, Monteverde A, et al. Cryoglobulinaemias: a multi-centre study of the early clinical and laboratory manifestations of primary and secondary disease. GISC. Italian Group for the Study of Cryoglobulinaemias. QJM Mon J Assoc Physicians 1995;88:115-26. 
[16] Terrier B, Krastinova E, Marie I, Launay D, Lacraz A, Belenotti P, et al. Management of noninfectious mixed cryoglobulinemia vasculitis: data from 242 cases included in the CryoVas survey. Blood 2012;119:5996-6004. doi:10.1182/blood-2011-12-396028.

[17] Terrier B, Semoun O, Saadoun D, Sène D, Resche-Rigon M, Cacoub P. Prognostic factors in patients with hepatitis $C$ virus infection and systemic vasculitis. Arthritis Rheum 2011;63:1748-57. doi:10.1002/art.30319.

[18] Terrier B, Karras A, Kahn J-E, Le Guenno G, Marie I, Benarous L, et al. The spectrum of type I cryoglobulinemia vasculitis: new insights based on 64 cases. Medicine (Baltimore) 2013;92:61-8. doi:10.1097/MD.0b013e318288925c.

[19] Fermand J-P, Bridoux F, Kyle RA, Kastritis E, Weiss BM, Cook MA, et al. How I treat monoclonal gammopathy of renal significance (MGRS). Blood 2013;122:3583-90. doi:10.1182/blood-2013-05-495929.

[20] Sidana S, Rajkumar SV, Dispenzieri A, Lacy MQ, Gertz MA, Buadi FK, et al. Clinical presentation and outcomes of patients with type 1 monoclonal cryoglobulinemia. Am J Hematol 2017;92:668-73. doi:10.1002/ajh.24745.

[21] Harel S, Mohr M, Jahn I, Aucouturier F, Galicier L, Asli B, et al. Clinico-biological characteristics and treatment of type I monoclonal cryoglobulinaemia: a study of 64 cases. Br J Haematol 2015;168:671-8. doi:10.1111/bjh.13196.

[22] Cacoub P, Desbois AC, Comarmond C, Saadoun D. Impact of sustained virological response on the extrahepatic manifestations of chronic hepatitis C: a meta-analysis. Gut 2018. doi:10.1136/gutjnl-2018-316234.

[23] Saadoun D, Pol S, Ferfar Y, Alric L, Hezode C, Si Ahmed SN, et al. Efficacy and safety of sofosbuvir plus daclatasvir for treatment of $\mathrm{HCV}$-associated cryoglobulinemia vasculitis. Gastroenterology 2017;153:49-52.e5. doi:10.1053/j.gastro.2017.03.006. 
[24] Cacoub P, Buggisch P, Carrión JA, Cooke GS, Zignego AL, Beckerman R, et al. Direct medical costs associated with the extrahepatic manifestations of hepatitis $\mathrm{C}$ infection in Europe. J Viral Hepat 2018. doi:10.1111/jvh.12881.

[25] Sise ME, Bloom AK, Wisocky J, Lin MV, Gustafson JL, Lundquist AL, et al. Treatment of hepatitis $\mathrm{C}$ virus-associated mixed cryoglobulinemia with direct-acting antiviral agents. Hepatol Baltim Md 2016;63:408-17. doi:10.1002/hep.28297.

[26] Terrier B, Krastinova E, Marie I, Launay D, Lacraz A, Belenotti P, et al. Management of noninfectious mixed cryoglobulinemia vasculitis: data from 242 cases included in the CryoVas survey. Blood 2012;119:5996-6004. doi:10.1182/blood-2011-12-396028.

[27] Terrier B, Marie I, Launay D, Lacraz A, Belenotti P, de Saint-Martin L, et al. Predictors of early relapse in patients with non-infectious mixed cryoglobulinemia vasculitis: Results from the French nationwide CryoVas survey. Autoimmun Rev 2014;13:630-4. doi:10.1016/j.autrev.2013.11.006.

[28] Dammacco F, Sansonno D. Therapy for hepatitis C virus-related cryoglobulinemic vasculitis. N Engl J Med 2013;369:1035-45. doi:10.1056/NEJMra1208642. 
Table 1. Main clinical, laboratory, and associated manifestations according to the immunochemical type of cryoglobulinemia $[16,18,20,28]$

\begin{tabular}{|lcc|}
\hline Immunochemical type & $\mathbf{I}^{\mathbf{a}}$ & II-III $^{\mathbf{b}}$ \\
& & \\
-- & & \\
Skin & & \\
Purpura & $25-40 \%$ & $75-90 \%$ \\
Raynaud's phenomenon & $25 \%-40 \%$ & $20-30 \%$ \\
Distal ulcers/necrosis & $30-35 \%$ & $5-15 \%$ \\
Cold-induced symptoms/cold urticaria & $90-100 \%$ & $0-10 \%$ \\
Livedo & $10-15 \%$ & $5-10 \%$ \\
Arthralgia/arthurite & $25-30 \%$ & $50-80 \%$ \\
Neurological involvement & $30-50 \%$ & $50-75 \%$ \\
Peripheral neuropathy & exceedingly rare & $5-10 \%$ \\
CNS involvement & $15-30 \%$ & $30-40 \%$ \\
Renal involvement & exceedingly rare & rare \\
Gastrointestinal involvement & exceedingly rare & rare \\
Cardiac involvement & exceedingly rare & rare \\
Pulmonary involvement & & \\
& & \\
\hline
\end{tabular}

CNS, central nervous system

${ }^{\text {a }}$ simple cryoglobulin composed of a monoclonal immunoglobulin

${ }^{\mathrm{b}}$ mixed cryoglobulins; type II: monoclonal and polyclonal immunoglobulins; type III:

polyclonal immunoglobulins only 
Table 2. Differences according to cryoglobulin type

\begin{tabular}{|l|c|c|}
\hline Mechanism & Type I cryoglobulin & $\begin{array}{c}\text { Mixed (type II/III) } \\
\text { cryoglobulin }\end{array}$ \\
\hline $\begin{array}{l}\text { Clinical } \\
\text { manifestations }\end{array}$ & $\begin{array}{c}\text { Occlusion of the capillary lumen } \\
\text { Vasculitis is uncommon. }\end{array}$ & $\begin{array}{c}\text { Small-vessel vasculitis++ } \\
\text { Occlusion of the capillary } \\
\text { lumen is less common. }\end{array}$ \\
\hline Laboratory tests & $\begin{array}{c}\text { Cold-induced symptoms } \\
\text { HF activity is rare. } \\
\text { Hypocomplementemia is } \\
\text { inconsistent. }\end{array}$ & $\begin{array}{c}\text { Purpura, arthralgia, } \\
\text { glomerulonephritis }\end{array}$ \\
\hline $\begin{array}{l}\text { Type of } \\
\text { monoclonal Ig }\end{array}$ & IgM $>\operatorname{IgG}>\operatorname{IgA}$ & $\begin{array}{c}\text { RF activity } \\
\text { C4 consumption }\end{array}$ \\
\hline
\end{tabular}

RF, rheumatoid factor; Ig, immunoglobulin; C4, complement component 4 
Box 1. Manifestations associated with cryoglobulin production; the most common are in bold type

\section{B-CELL MALIGNANCIES}

Waldenström macroglobulinemia

Multiple myeloma (Plasmacytoma)

MGUS

B-cell non-Hodgkin's lymphoma

Chronic lymphocytic leukemia

Hairy-cell leukemia

\section{SYSTEMIC and/or AUTOIMMUNE DISEASES}

\section{Sjögren's syndrome}

Systemic lupus erythematosus

Rheumatoid arthritis

Schönlein-Henoch purpura

Dermatopolymyositis

Scleroderma

Granulomatosis with polyangiitis (e.g., Wegener granulomatosis)

Periarteritis nodosa

Behçet's disease

Sarcoidosis

Autoimmune thyroiditis

Primary biliary cirrhosis

Autoimmune hepatitis

Celiac disease

Pemphigus vulgaris

Endomyocardial fibrosis

Idiopathic pulmonary fibrosis

\section{INFECTIONS}

\section{Viral infections}

Chronic hepatitis C

Chronic hepatitis B

Epstein-Barr virus infection

Cytomegalovirus infection

Acute hepatitis A

Human immunodeficiency virus infection

Adenovirus infection

Parvovirus B19 infection

\section{Bacterial infections}

Subacute endocarditis

Syphilis

Acute post-streptococcal glomerulonephritis

Lyme disease

Brucellosis

Coxiella infection

Mediterranean spotted fever

Atrioventricular shunt infection

Lepromatous leprosy 
Parasitic infections

Paludism

Visceral leishmaniasis

Toxoplasmosis

Schistosomiasis

Echinococcosis

Tropical splenomegaly syndrome

Fungal infections

Coccidioidomycosis

4. OTHER

Extracapillary glomerulonephritis

Cancer: breast, nasopharynx, esophagus 
Figure 1: Clinical manifestations of cryoglobulinemic vasculitis
a. Severe skin ulcer
b. Nerve biopsy specimen showing vasculitis with a perivascular inflammatory infiltrate
c. Distribution of the peripheral neurological involvement indicating length dependency
d. Renal biopsy showing membranoproliferative glomerulonephritis
e. Magnetic resonance imaging of the brain showing vasculitis

\section{Figure 2: Diagnostic strategy for cryoglobulinemia}

Determine the type of cryoglobulin

Type I cryoglobulinemia

Mixed cryoglobulinemia

IgM++
B-cell non-Hodgkin's
lymphoma (Waldenström,
marginal zone...)

$\operatorname{IgG}>\operatorname{Ig} \mathrm{A}$

Myeloma

Hepatitis C

B-cell non-Hodgkin's

Connective tissue disease

lymphoma

(Sjögren's, lupus)

MGUS

B-cell lymphoma

Other 


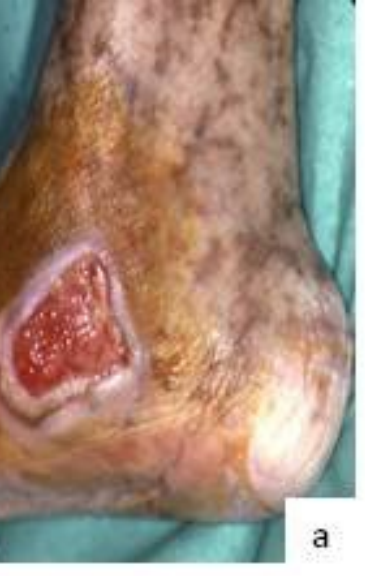




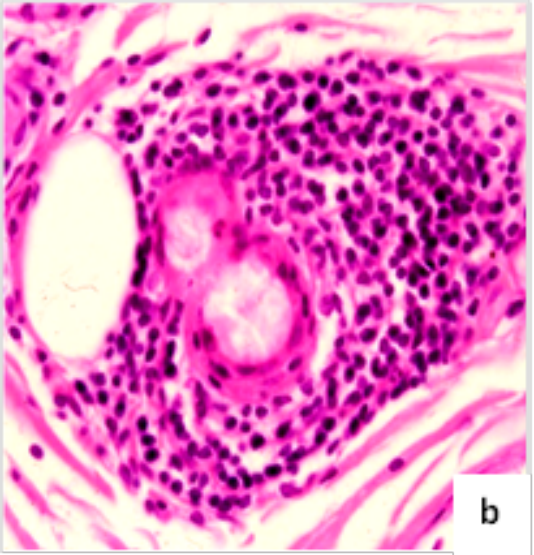




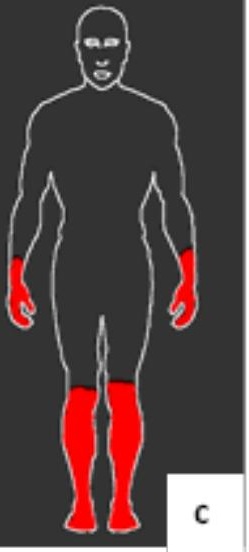




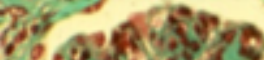

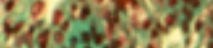

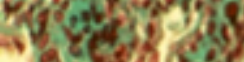

b.

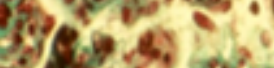

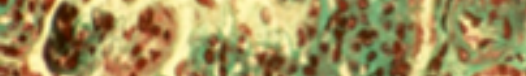

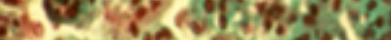

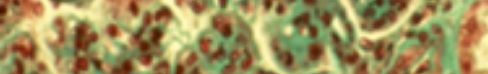

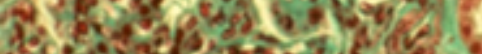

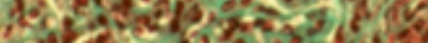
is

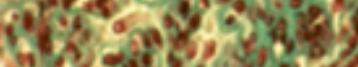

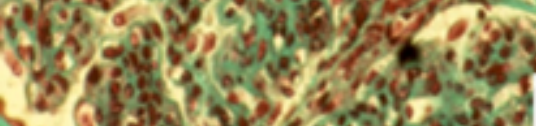

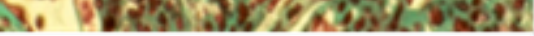
2. (a)

$$
\operatorname{lig}^{2} \frac{2}{8}
$$

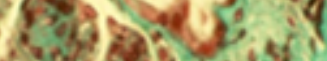

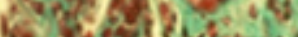

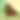
20 
30122

finteros

in 940.

R
9
9

$\varepsilon$

thister

tifere

EC:2/2 ar:

Mond

Foriscon

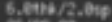

3rens

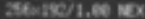
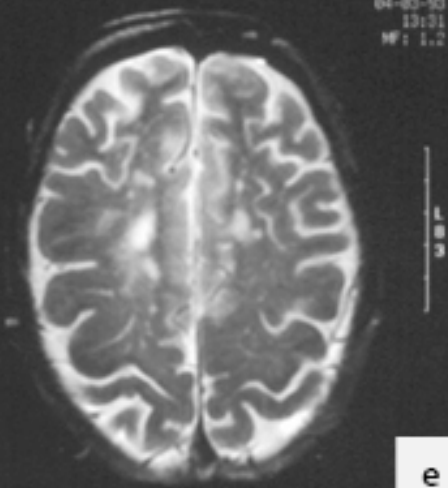

e this wi is

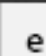




\section{Typage cryoglobuline}

\section{Cryoglobulinémie de} type 1

lgM++

Lymphome non hodgkinien B

(Waldenstrom, zone marginal...)

$\lg \mathrm{g}>\lg A$
Myélome

Lymphomenon

Hodkinien B

MGUS

Cryoglobulinémie mixte

Hépatite C

Connectivite (Gougerot, Lupus)

Lymphome B

\section{Autres}

